\title{
Review on Molecular Components for Electronic Circuits
}

\author{
Deep Kamal Kaur Randhawa \\ Department of Electronics \& Communication Engineering, \\ Guru Nanak Dev University Regional Campus, Jalandhar, India
}

\begin{abstract}
Electronics has been the driving force behind the technical growth for the second half of twentieth century. The evolution of computational techniques and communication technologies has been result of rapidly developing silicon integrated circuit industry. Reduction of size of transistor leading to denser and faster IC's has been governed by the famous Moore's Law. The silicon is now approaching its physical limitations in terms of reduction of size and also the laws of physics governing small sizes.The trend is pushing electronics towards the realm of molecular electronics. Molecules are small in size and exhibit properties of self-assembly and selfrecognition. These properties render the molecules to be highly useful for the bottom-up nanotechnology. Exact chemical equivalence of the molecules would provide similar electronic devices. Last but not the least Molecular Electronics will provide us with cooler laptops and mobile phones.
\end{abstract}

\section{Keywords}

Molecular Electronics, Self-assembly, Self-recognition, Bottom-up technology.

\section{INTRODUCTION}

The legacy of semiconductor electronics has ruled the modern lifestyle in a big way. The next big thing to happen is small.... very small: Molecular Electronics. Molecular microchips populated by molecular transistors will be faster, cheaper and energy saving. They will transcend all the limitations of magnetic and optical storage technologies. As silicon is approaching its fundamental limits, it can be said that Silicon Valley will soon have a sibling called Molecular Valley. Molecular electronics can be viewed as the design, fabrication and assembly of molecules into functioning electronic circuits.

\footnotetext{
Molecular electronics can compress the fabrication process into a matter of few hours, all thanks to self-assembly property of the molecules. Aviram says, "With molecular electronics, you can build your transistors in a pot, a year's supply at a time." Molecular electronics (also known as "moletronics") is the promising current endeavor in the field of nanotechnology, which engross the investigations related to the electronic structure, electron transport and device exploitation of matter at their molecular degree [1]. Presently molecular electronics is the growing field of interest and lots of experimental and theoretical research activities are going on worldwide. A look at the present mushrooming stage of molecular electronics shows that the science fiction is marching towards the reality to explain how a hefty amount of data can be placed over the head of a small pin.
}

Since the molecules are very small, their functionality can be tuned. Development of molecular and nanoelectronic components such as wires, diodes, transistors, oscillators and switches [2],[3] as well as the conceptual discussion of their current-voltage properties have greatly enhanced the zeal for designing novel molecular systems with typical electronic properties. Several attempts have been made to theoretically explain the current-voltage characteristics of the molecular systems [4],[5]. There are various candidates for molecular devices such as- organic polymers [6],[7], large bio-molecules [8],[9], nanotubes \& fullerines [10],[11].

The distinguishing features other than small size, which favor the use of molecules as electronic components, are the band structure, fexible nature of molecules and delocalisable $\mathrm{p}$ systems. Property of exact chemical equivalence and self assembly by organic molecules further supports the use of these molecules as they will be helpful in manufacturing large arrays of identical devices in a defect free fashion.

\section{MOLECULAR ELECTRONIC DEVICES}

Semiconductor electronics, the backbone of the communication and computational technology is based on integration of various electronic devices like transistors, diodes and resistors connected through wires [12]. The typical current-voltage characteristics of the devices are exploited to manipulate the inputs to customize the outputs. Transistor is the fundamental building block of semiconductor electronics that are used to control the flow of current from source to drain.

As electronics moves towards nanometer range the magnitude of the current flowing through the devices is also decreased. The ever reducing sizes and current is taking electronic devices into the domain of single electron effects. So while studying use of molecules as electronic devices single electron effects have to be discussed too.

\subsection{Molecular Wires}

Wire is one of the most essential parts of an electronic circuit that provides a path for transport of electric charge [13]. At molecular level, wire would be a structure that transports electrons from one end to other [14]. It is expected that electron transport will be through the frontier molecular orbitals. So large delocalized $\pi$-systems with reduced HOMOLUMO Gaps (HLG) can be considered as promising candidates for molecular wires. Due to very small HLG, the process is thermodynamically favorable and ultimately gives rise to efficient wire function. The simplest chain for use as wire is polyene consisting of alternating sequence of single and double bond forming a $\pi$-system. Aromatic building blocks like polybenzene, combination of aromatic building blocks with conjugated double or triple bonds like polyphenylenevinylene [15] are a few examples (structures 
shown in Fig. 1) which have been studied extensively for use as molecular wire.

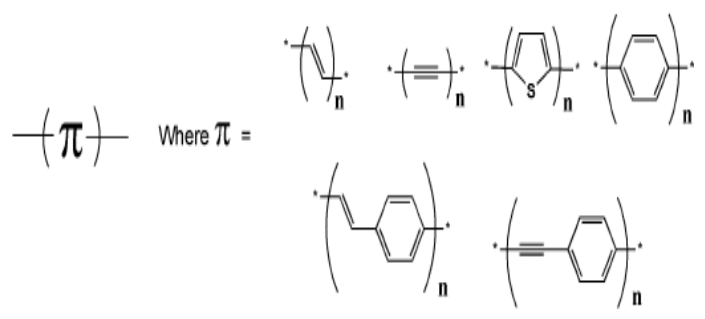

Fig. 1 Molecular Structures acting as Wires

In context with organic molecules it can be said that the the pi-type systems mimic wire function and studies related to similar type of molecular wires are also described in the literature [16]-[19].

\subsection{Molecular Resistor}

An electronic circuit designer is not always interested in property of conductance but also in the opposite property called resistor. The insulating properties of material are also of equal significance for design of analog and digital circuits. Resistance basically displays the characteristic of material which opposes flow of electrical charge. From the literature it can be seen that, most widely investigated organic molecules to achieve resistor type of behavior, are as Fig.2. [20],[21]

Aviram and Ratner suggested a rigid adamantly cage as a nonconjugating linker between two $\pi$-systems and it was expected to behave as an insulating molecular unit.The spacer should be sufficiently insulating so as to preserve the energy difference between the $\pi$-systems while allowing limited electron transport by tunneling. Alkanes are known to display insulating properties. In these molecules presence of $-\mathrm{CH}_{2}-$ units create nodes in the electron densities above nuclei. For this reason and also due to large HLG, the aliphatic molecules behave as resistors. The molecules without delocalized $\pi$ systems boast of excellent insulating properties.
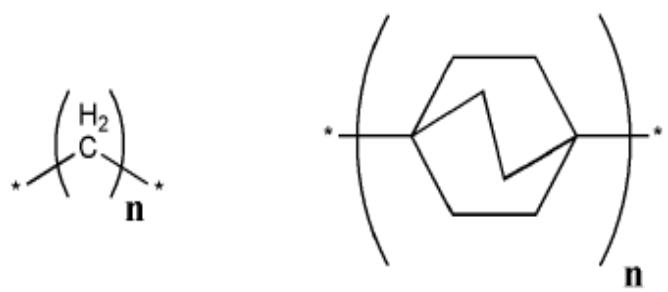

Fig. 2 Molecular Structures acting as Resistors

\subsection{Molecular Diode}

The function of diode is one of the major aspects of molecular electronics. A diode is a unilateral device which ideally conducts in one direction only and is very widely used as a rectifier. Molecular diode can be visualized as an assembly of a molecule inserted between a pair of metallic electrodes (Fig 3 ), performing function of a rectifier [22].

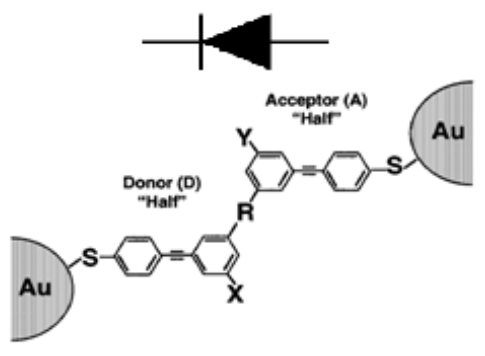

Fig 3. Donor-Spacer-Acceptor Structure

The suggested operation of rectifier is based on the principle that there is difference in energy of the frontier molecular orbitals of the 'donor-' and 'acceptor-' ' $\pi$ - systems'. The spacer preserves the energy difference between the donor and acceptor $\pi$ - systems, but allows electron transport to certain extent. The unequal difference of energy levels of frontier molecular orbitals w.r.t. equilibrium energy levels of the metal electrodes as shown in Fig.4 result in preferential direction of flow of current on application of terminal potential. This behavior of $\mathrm{D}-\sigma-\mathrm{A}$ assembly can be regarded as rectifier function. The acceptor part of the molecule considered as p-type semiconductor while donor part can be regarded as n-type, while the spacer can be compared to the junction barrier. Rectification using cumulenic bridges [23] suggests the use of cumulenic compounds due to their synthetic feasibility [24] and structural rigidity. Also the material used for electrodes will also affect the performance of molecular diodes.
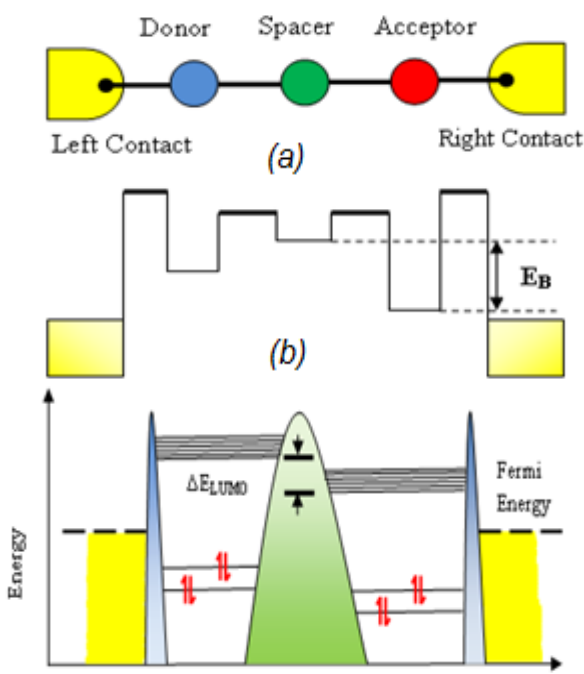

(c)

Fig 4. a) Contact-D-o-A-Contact Assembly b) Differential Energy levels c) Frontier molecular orbital representation of $\mathrm{D}-\sigma-\mathrm{A}$, with $\Delta \mathrm{E}_{\mathrm{LUMO}}$ represents the barrier.

Reed and Tour [25] proposed another interesting device based on rod-like molecule inserted between two gold electrodes. This device not only displayed diode like characteristics but also exhibited Negative Differential Resistance (NDR) in the current profile. For a certain section of voltage sweep the 
current reduces for increasing voltage displaying negative resistance. This effect could be exploited in molecular electronics for design of a different class of diodes called Resonant Tunneling Diodes (RTD). RTD's are studied widely because of their potential use in very high speed/functionality circuits. It is a promising nanoelectronic device for both analog and digital applications. The operation of RTD (Fig 5) is based on resonant tunneling of electrons through the 'island molecule' whenever electrochemical potential of the electrodes is aligned with the discrete energy levels of island. So the device is ON whenever kinetic energy of electrons is equal to that of the energy levels of island and is OFF whenever the energy is different [26].

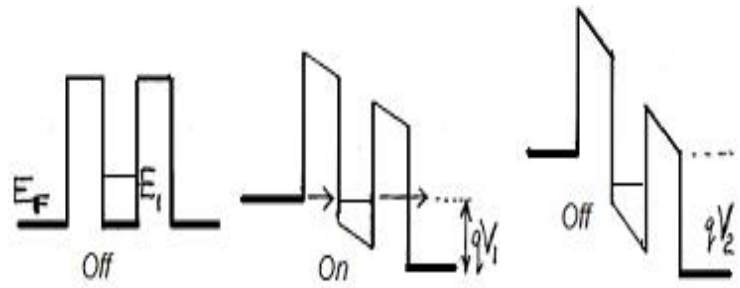

Fig 5. Resonant Tunneling Diode

\subsection{Three Terminal Devices}

Three terminal devices are the integral element of an electronic circuit as they provide power amplification to the signal. To achieve functional equivalence to semiconductor electronic circuits, realization of molecular three terminal devices (Fig.6) is of utmost importance. The main hiccup in realization of three terminal devices is structuring three independent nanometer sized leads.

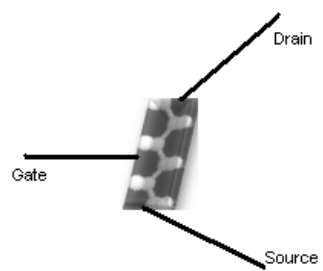

\section{Fig.6 Molecular Three Terminal Device}

There are two possible approaches to realize the three terminal molecular devices. First is to assemble a molecule with three ports, where each port would be connected to an independent electrode. The other approach is to attach the third lead at a distance form the two port molecule so that it does not have direct contact with the molecule. The potential applied on the third terminal can be used to alter the electrostatic state of molecule by field effect. This has been demonstrated [27], [28] at low temperatures. The tuning of conductivity of orgnometallic molecules implies presence of Single Electron Effects, thus suggesting use of molecules for designing molecular single electron devices. The gate potential can be used to switch the single electron transistor from coulomb blockade regime to conducting mode.

\subsubsection{Molecular Transistor}

Molecular transistor has a molecule inserted between two metallic/semiconducting terminals. The third electrode called gate is electrostatistically coupled to the molecule as shown in
Fig. 7 a) and b). The HOMO-LUMO gap acts as barrier for conduction of electrons. As shown in Fig 7(c). The Fermi energy of the electrodes lies in the middle of HLG of the molecule (no conduction).Application of bias across the metal-molecule-metal assembly disturbs the equilibrium energy levels. When the Fermi energy of the electrodes aligns with energy levels of the molecular orbitals (MO's) of the molecule (resonance of levels) the current will flow, irrespective of the fact that the orbitals are occupied or not. If the occupied MO's participate in the conduction then it called p-type of conduction. Flow of charge carriers through unoccupied MO's is known as n-type of conduction. The energy levels of the molecule can be elevated or lowered depending upon the polarity of Gate bias. The shifting of MO's change the status of MO's participating in conduction, thus controlling the flow of current.

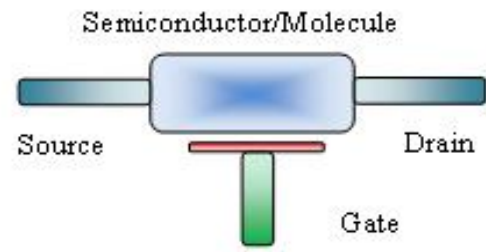

(a)

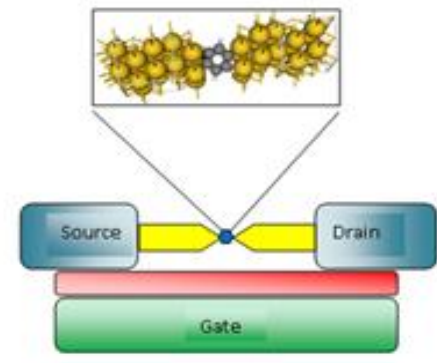

(b)

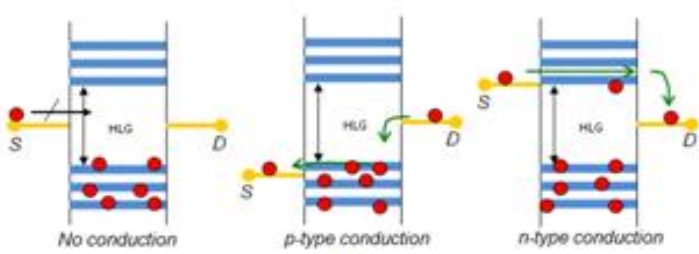

(c)

Fig 7. a),b)Molecular Transistor c) Schematic of electron flow through Molecular Transistor

2.4.2 Molecular Single Electron Transistor The geometry of molecular Single Electron Transistor consists of metallic source and drain electrodes, and a molecular island coupled with the two electrodes (Fig 8). Electrons can propagate from source to drain through the island. If the island is strongly coupled with the source and drain electrodes, the electrons will stay a very short time on the island, and cannot localize but will move coherently through the system. This is the regime where we can use the coherent transport model for simulating the electrical properties of the system. 


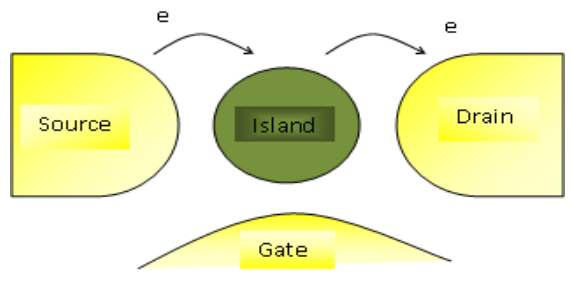

Fig.8 Schematics of Single Electron Transistor. Electrons propagate from the source to the drain through an island. The energies of the electronic states on the island can be controlled by an electrostatic gate.

As the small size of molecules is very small, the energy required to move an electron onto the island from source is very large. This leads to digitization of charging in the device leading to single electron effects exhibiting coulomb staircase and oscillations phenomena.

\subsection{Switches and Storage Elements}

The property of certain molecules to exist in two different stable states having different conductive properties leads us to the idea for using these molecules as storage elements. Bistable molecular switches can be made using (i) redox (reduction-oxidation) process, (ii) configuration change through reversible re-arrangement reactions, (iii) conformation change if both confirmations are stable at operating temperature, (iv) electronically excited states and (v) use of spin magnetic moments [29].

In molecular bistable switches sometimes more than one type of mechanisms must co-operate to achieve bistable hysteresis. The switches are classified as into two sections based on (a) the triggering stimulus and (b) the property or function that is switched. The two stable states of the molecules can be used to depict two logic states $0 \& 1$, the binary logic states. These type of molecules can be used to make molecular memory cells in which each bistable state corresponds to the coding of a piece of binary information i.e. 0 or 1 . Rotaxanes and catenanes have been synthesized to switch as a function of applied potential between two states.

\section{CONCLUSION}

The future is getting smaller yet powerful. Apart from organic molecules, there are many potential molecules available which can be explored for Molecular electronics. DNA, carbon nanotubes, proteins etc are possible candidates that can be scaled to very small sizes and designed to accomplish specific tasks. This will be huge leap in technology using tiny molecules. The need of hour is to model robust methods for fabrication processes that can be used to replicate the achievements of lithographic processes. Attaining control over the properties of metal-molecule or molecule-molecule interfaces would further help in creating molecular integrated circuits. This achievement would pave a path towards interfacing biological systems with ultra-dense molecular integrated circuitry.

\section{REFERENCES}

[1] M. A. Reed, T. Lee (Eds.) "Molecular Nanoelectronics", American Scientific Publishers, Stevenson Ranch, CA, 2003.

[2] C.W. Bauschlicher Jr., J.W Lawson, "Current-voltage curves for molecular junctions: the issue of the basis set for the metal contacts" Phys. Rev. B 75 115406115411,March 2007

[3] S.Datta, "Electronic Transport in Mesoscopic Systems", Cambridge University Press, New York, 1996.

[4] Y.-h. Zhou,X.-h. Zheng, Y.Xu, Z.Y.Zeng, “ Current Rectification by asymmetric molecules: An ab initio study" J.Chem. Phys. 125 244701-244705,December 2006.

[5] M.Di Ventra, S.T. Pantelides, N.D. Lang, "FirstPrinciples Calculation of Transport Properties of a Molecular Device “, Phys. Rev. Lett. 84 979-982, January 2000

[6] Aviram A, Ratner MA, "Molecular rectifiers", Chem Phys Lett 29, 277-283(1974)

[7] Collier CP et. al. "A [2]Catenane-Based Solid State Electronically Reconfigurable Switch", Science 289:1172-75(2000)

[8] Keren $\mathrm{K}$ et. al. "Sequence-specific molecular lithography on single DNA molecules", Science 297:72-75(2002)

[9] Porath D et.al., "Direct measurement of electrical transport through dna molecules",Nature 403:635$38(2000)$

[10] Rinaldi R et. al. "Transport in hybrid electronic devices based on a modified DNA nucleoside (deoxyguanosine)", Annals of the New York Academy of Sciences 960:184-192(2002)

[11] Benenson Y et.al. "DNA molecule provides a computing machine with both data and fuel" Proc Natl Acad Sci USA 100:2191-96(2003)

[12] M. D. Ward, " Chemistry and Molecular Electronics: New Molecules as Wires, Switches, and Logic Gates", J. Chem. Edu., 78, 321-28, March 2001

[13] W.B Davis, et. al., "Molecular-Wire Behaviour in pPhenylenevinylene Oligomers" Nature 396, 60-63, November 1998.

[14] J.M. Tour, "Conjugated Macromolecules of Precise Length and Constitution. Organic Synthesis for the Construction of Nanoarchitectures", Chem, Rev. 96,53754, February 1996.

[15] R. Waiser, “ Nanoelectronics and Information Technology- Advanced Electronic Materials and Novel Devices", Wiley-VCH, 504-5,2005.

[16] N . Robertson, C. A. MaGowan, "A comparison of potential molecular wires as components for molecular electronics" Chem. Soc. Rev., 32, 96-103, 2003.

[17] R. L. Carroll, C. B. Gorman, "The Genesis of Molecular Electronics", Angew. Chem. Int. Ed. Engl., 41, 43784400,November 2002.

[18] D. K. James, J. M. Tour, "Molecular Wires" in Encyclopedia of Nanoscience and Nanotechnology, Marcel Dekker, New York, 2177-95, 2004

[19] I. Alkorta, J. Elguero, "Polyynes vs. Cumulenes: Their Possible Use as Molecular Wires",Structural Chemistry, 16, 77-79, February 2005 
[20] N. Jonassen, Surface Voltage and Field Strength: Part I, Insulators, in Mr.Static, Compliance Engineering 18, 26, 2001.

[21] N. Jonassen, Abatement of Static Electricity: Part II, Insulators, in Mr.Static, Compliance Engineering 18, 26, 2001.

[22] A. Aviram and M. Ratner, "Molecular Rectifiers", Chem. Phys. Lett. 29, 277-83 November 1974

[23] Sanyasi Sitha, K. Bhanuprakash, B.M.Choudary, "Electrical Rectification through Cumulenic Bridge: A Computational Study", Synthetic Metals 148, 227-235, February 2005.

[24] L.Brandsma, Synthesis of Acetylenes, Allenes and Cumulenes, Academic Press, Orlando,2003
[25] J. Chen et al , "Large On-Off Ratios and Negative Differential Resistance in Molecular Electronic Device",Science 286, 1550-52, November 1999.

[26] M. Jagadesh Kumar, “ Molecular Diodes and Applications", Recent Patents on Nanotechnology ,1, 5157,February 2007

[27] J.Park et al , "Coulomb Blockade and the Kondo Effect in single -atom transistors", Nature 417,722-25,April 2002.

[28] W.Liang,et al, "Kondo resonance in a single-molecule transistor", Nature,417, 725-29, June 2002

[29] R.Sessoli et al, "Magnetic bistability in a metal-ion cluster", Nature 365, 141-43, September 1993. 